\title{
Non-Recurrent Behaviour in Quantum Dynamics
}

\author{
Giulio Casati ${ }^{1}$ and Italo Guarneri ${ }^{2}$ \\ 1 Dipartimento di Fisica dell'Università di Milano, Via Celoria 16, I-20133 Milano, Italy \\ 2 Dipartimento di Fisica Nucleare e Teorica dell'Università di Pavia, Via Bassi, I-27100 Pavia, \\ Italy
}

\begin{abstract}
We study the motion of a quantum rotator under an external periodic perturbation. For the resonant case, i.e. when the frequency of driving pulses is rationally connected with the frequencies of the free rotator, the quasi-energy spectrum is known to be continuous. We prove that for a generic choice of the potential there is a non-empty set of non-resonant values of the external frequency such that the quasi-energy spectrum still has a continuous component.
\end{abstract}

I.

Up to now, quantum mechanics has offered but a poor surrogate for the variety of the forms in which chaotic behaviour can manifest in classical mechanics. Discreteness of the spectra of bounded systems and localization of states in unbounded systems with disordered potentials are major drawbacks that seem to constrain the search for chaotic quantum motion to the field of time-dependent Hamiltonians, and to time-periodic Hamiltonians in particular.

The so-called " $\delta$-kicked rotator" has been being used extensively as a model system in this line of research ${ }^{[1-7]}$. It displays non-recurrent behaviour, associated with a continuous component in the quasi-energy spectrum, when the frequency of the driving pulses is rationally connected with the frequencies of the free rotator ${ }^{[2]}$. However interesting in itself, this "quantum resonance" is quite another type of behaviour than chaotic; therefore, the latter can possibly take place only for "nonresonant" values of the driving frequency.

For such values, numerical evidence is that there are strong limitations to the occurrence - if any - of non-recurrent behaviour ${ }^{[1-3]}$. These desultory numerical results, together with the recently discovered similarity of the rotator problem with the problem of localization ${ }^{[6]}$, seem to support the thesis that there are no possibilities for periodically driven quantum systems other than recurrence and resonance.

The result we present in this paper is that a "third way" actually exists. In fact, we prove that for a "generic" choice of the potential of the kicked rotator there are 
non-resonant situations in which the quasi-energy spectrum still has a continuous component; also, there are reasons to conjecture that this component is actually a singular continuous one.

We give the proof in Sect. II. In the conclusive Sect. III we discuss somewhat the relevance of this result, as well as some possibilities of future development.

II.

We study the quantum properties of the $\delta$-kicked rotator, described by the Hamiltonian:

$$
\hat{H}=-\frac{1}{2} \frac{d^{2}}{d \theta^{2}}+\mu V(\theta) \sum_{n=-\infty}^{\infty} \delta(t-4 \pi n \tau),
$$

where $V(\theta)$ is an analytical, real-valued, periodic function of $\theta$, and we have assumed $\hbar=1$. We denote by $C$ the unit circle.

Mapping over one period involves free rotation and a kick:

$$
\begin{aligned}
(S \psi)(\theta) & =e^{-i \mu V(\theta)} \sum_{-\infty}^{\infty} c_{n} e^{-i\left(2 \pi \tau n^{2}-n \theta\right)} \\
\psi(\theta) & =\sum c_{n} e^{i n \theta} .
\end{aligned}
$$

From (1) we see that it suffices to consider values of $\tau$ within the interval $[0,1]$.

Let us consider first the "resonant" case - in which $\tau=p / q, p, q$ integers. To $\psi \in \mathscr{L}^{2}(C)$, we associate the $q$-vector

$$
\left(\psi(\theta), \psi\left(\theta+\frac{2 \pi}{q}\right), \ldots, \psi\left(\theta+\frac{2 \pi(q-1)}{q}\right)\right), \quad 0 \leqq \theta<\frac{2 \pi}{q} .
$$
In this way $\mathscr{L}^{2}(C)$ is identified with $\mathscr{L}^{2}\left(I_{q}, C^{q}\right)$, where $I_{q}=\left(0, \frac{2 \pi}{q}\right)$. As shown in
ref. (2), in this case $S$ allows for the integral decomposition

$$
S=\int_{0}^{2 \pi / q} d \theta S_{\theta}
$$

with $S_{\theta}$ a $q \times q$ unitary matrix: $S_{\theta}=W_{\theta} \Gamma . W_{\theta}$ is a diagonal matrix,

$$
\left(W_{\theta}\right)_{n m}=\delta_{n m} e^{-i \mu V\left(\theta+\frac{2 \pi n}{q}\right)}(0 \leqq n, m \leqq q-1),
$$

and $\Gamma$ is a circulant matrix independent of $\theta$. We are not interested in further specification of $\Gamma$. For more details, see ref. (2).

$S_{\theta}$ has eigenvalues depending on $\theta$, and, as shown in [2], at least one of them is not constant over $I_{q}$ : this eigenvalue originates a continuous band in the spectrum of $S$.

We are interested in $S$ having a purely continuous spectrum, i.e., no eigenvalues. Eigenvalues of $S$ occur when $S_{\theta}$ has constant eigenvalues over sets of positive measure in $I_{q}$. Since $S_{\theta}$ is a matrix-valued analytic function of $\theta$, its eigenvalues will be analytic functions of $\theta$ - except for branch points at values where level crossing occurs. Therefore, if an eigenvalue $\lambda(\theta)$ is constant over a set of positive measure, it will be constant all over $I_{q}$. 
Then, consider the analytic, real-valued, periodic functions $V(\theta)$ in $C$, and let $\mathscr{B}_{\alpha}$ be the vector space of all such functions having an analytic continuation in the strip $C_{\alpha}=\{|\operatorname{Im} z|<\alpha\}$, continuous in $\bar{C}_{\alpha} . \mathscr{B}_{\alpha}$ is a Banach space with the norm $\|\psi\|_{\alpha}=\max _{z \in \bar{C}_{\alpha}}|\psi(z)|$.

Theorem 1. For any $\alpha>0$, the set of $V \in \mathscr{B}_{\alpha}$ such that $S$, in resonance, has a purely continuous spectrum contains a set of the second Baire category in $\mathscr{B}_{\alpha}$. In this sense we'll say that generic analytic $V$ have this property.

Proof. Suppose that $\lambda$ is a constant, nondegenerate eigenvalue for a given $\tau=p / q$, and let $x=x(\theta)$ be a corresponding eigenvector ( $q$-vector). Then $S_{\theta} x=\lambda x$, whence, differentiating,

$$
\dot{S}_{\theta} x+S_{\theta} \dot{x}=\lambda \dot{x} \text {, i.e. }\left(\lambda-S_{\theta}\right) \dot{x}=\dot{S}_{\theta} x=\dot{W}_{\theta} \Gamma x=M_{\theta} S_{\theta} x=\lambda M_{\theta} x,
$$

where $\left(M_{\theta}\right)_{m n}=-i \mu V^{\prime}\left(\theta+\frac{2 \pi m}{q}\right) \delta_{m n}$. Therefore, we must have $\left(M_{\theta} x, x\right)=0$ for all $\theta \in I_{q}$; in particular,

$$
\sum_{j=0}^{q-1} V^{\prime}\left(\frac{2 \pi j}{q}\right)\left|x_{j}(0)\right|^{2}=0
$$

The matrix $S_{0}$ is fixed by the specification of the $q$ numbers $V_{j}=V\left(\frac{2 \pi j}{q}\right)$; therefore the left-handside of (2) defines a function of the $2 q$ numbers: $V_{j}, V_{j}^{\prime}=V^{\prime}\left(\frac{2 \pi j}{q}\right)$.

In a neighbourhood of any point $\left(V_{j}, V_{j}^{\prime}\right)$ corresponding to a matrix with no degenerate eigenvalues, this function is actually a continuous one. Therefore, the set of values $\left(V_{j}, V_{j}^{\prime}\right)$ such that

(i) $S_{0}$ has no degenerate eigenvalues,

(ii) Condition (2) is not satisfied by any eigenvector of $S_{0}$,

is open in $C^{2 q}$, being the intersection of the open sets defined by (i) and (ii) separately; the set $X_{p, q, \alpha}$ of $V \in \mathscr{B}_{\alpha}$ such that (i) and (ii) are satisfied by $\left(V_{j}, V_{j}^{\prime}\right)$ is likewise open, because the map from $\mathscr{B}_{\alpha}$ into $C^{2 q}$ defined by $V(\theta) \rightarrow\left(V_{j}, V_{j}^{\prime}\right)$ is continuous, as a consequence of Cauchy's formula. This set is dense, also. In fact, given $V \in \mathscr{B}_{\alpha}$, we can first remove any degeneracy from the corresponding $S_{0}$ by an arbitrarily small variation of $V$. (This we prove in the Appendix.) If the $\bar{S}_{0}$ thus obtained satisfies (2) for one of its eigenvectors, then we may change $V(\theta)$ into $\bar{V}+\varepsilon \sin q \theta=\overline{\bar{V}}(\theta) \cdot \overline{\bar{V}}(\theta)$ is arbitrarily close to $\bar{V}(\theta)$ in $\mathscr{B}_{\alpha}$ and its matrix $S_{0}$ has the same eigenvectors, but (2) is no longer satisfied.

Consider now the set $X_{\alpha}=\bigcap_{p, q} X_{p, q, \alpha}$. If $V \in X_{\alpha}$, then, for all $p, q, S_{0}$ will have no degenerate eigenvalues, and no eigenvector of $S_{0}$ will satisfy (2). Therefore, for any $\tau=p / q, S_{\theta}$ will have no constant eigenvalues. Thus, $X_{\alpha}$ is contained in the set of those $V \in \mathscr{B}_{\alpha}$ which have purely continuous spectra in resonance; on the other hand, $X_{\alpha}$ is a countable intersection of dense open sets in $\mathscr{B}_{\alpha}$, i.e., it is a set of the second Baire category in $\mathscr{B}_{\alpha}$. 
So, for rational $\tau$ and generic $V(\theta), S_{\tau}$ will have a purely continuous spectrum (from now on, the index of $S$ will specify the period). This entails that, $\forall \psi \in L^{2}(C)^{[8]}$,

$$
\lim _{N \rightarrow \infty} \mathscr{R}_{\tau}(N, \psi)=\lim _{N \rightarrow \infty} \frac{1}{N} \sum_{K=1}^{N}\left|\left(S_{\tau}^{K} \psi, \psi\right)\right|^{2}=0 .
$$

We shall restrict our attention to those $\psi \in L^{2}(\dot{C})$ which give a finite expectation value for the kinetic energy:

$$
K(\psi)=\pi \sum_{-\infty}^{\infty} n^{2}\left|c_{n}\right|^{2}<\infty .
$$

For $E>0$, we define $\sigma_{E}=\left\{\psi \in L^{2}(C),\|\psi\|=1, K(\psi) \leqq E\right\}$.

Now we set out to prove our main result. We begin with some lemmas.

Lemma 1. Let $S_{\tau}$ have purely continuous spectrum. Then $\mathscr{R}_{\tau}(N, \psi) \rightarrow 0$ as $N \rightarrow \infty$, uniformly with respect to $\psi \in \sigma_{E}$.

Proof. For a fixed $\tau$ the sequence $\mathscr{R}_{\tau}(N, \psi)$ is equicontinuous in $\psi \in \sigma_{E}$; indeed:

$$
\left|\mathscr{R}_{\tau}(N, \psi)-\mathscr{R}_{\tau}\left(N, \psi^{\prime}\right)\right| \leqq \frac{2}{N} \sum_{K=1}^{N}\left\{\left|\left(S_{\tau}^{K} \psi, \psi-\psi^{\prime}\right)\right|+\left|\left(S_{\tau}^{K} \psi-S_{\tau}^{K} \psi^{\prime}, \psi^{\prime}\right)\right|\right\} \leqq 4\left\|\psi-\psi^{\prime}\right\| .
$$

Moreover, $\sigma_{E}$ is a compact set in $L^{2}(C)$.

Lemma 2. Let $\psi$ be entire analytic, $V \in \mathscr{B}_{\alpha}, F(\psi)=\sum n^{2 r}\left|c_{n}\right|^{2} ;(r>0)$. Then $\exists C$ such that, $\forall N, F\left(S^{N} \psi\right) \leqq C N^{2 r}$.

Proof. $S^{N} \psi \in \mathscr{B}_{\alpha}, \forall N$. Therefore, by the Cauchy theorem the Fourier coefficients $c_{n}(N)$ of $S^{N} \psi$ and $W_{n}$ of $e^{-\imath \mu V}$ satisfy:

$$
q_{\beta}(N)=\sup _{n} e^{\beta|n|}\left|c_{n}(N)\right|<\infty, \quad \forall \beta \in[0, \alpha], \quad\left|W_{n}\right| \leqq d e^{-\alpha|n|} .
$$

Moreover, if $c_{n}^{\prime}(N)$ are Fourier coefficients of $T \psi(N)$ [the "free" evolution of $\psi(N)$ over one period]

$$
\begin{aligned}
\left|c_{n}(N+1)\right| & =\left|\sum_{K} W_{n-K} c_{K}^{\prime}(N)\right| \leqq \sum_{K}\left|W_{n-K}\right|\left|c_{K}^{\prime}(N)\right| \\
& =\sum_{K}\left|W_{n-K}\right|\left|c_{K}(N)\right| \leqq d q_{\alpha / 2}(N) \sum_{K} e^{-\alpha / 2|K|} e^{-\alpha|n-K|} \\
& \leqq \text { const } d q_{\alpha / 2}(N) e^{-(\alpha / 2)|n|} .
\end{aligned}
$$

(The const does not depend on $n, N$.)

Thus we may assume $q_{\alpha / 2}(N+1) \leqq$ const $q_{\alpha / 2}(N)$, and $\left|c_{n}(N)\right| \leqq b^{N} e^{-\alpha / 2|n|}$ for a suitable $b>0$. This means that the pattern of the $c_{n}^{\prime} s$ cannot "spread" in time faster than linearly; it is then easy to conclude that $F\left(S^{N} \psi\right)<$ const $N^{2 r}$.

Lemma 3. Let $\psi$ be an entire function with $\|\psi\|=1$. Then

$$
\left|\mathscr{R}_{\tau}(N, \psi)-\mathscr{R}_{\tau^{\prime}}(N, \psi)\right| \leqq\left|\tau-\tau^{\prime}\right| \beta N^{3}
$$

for a suitable $\beta>0$ independent of $\tau$ and $\tau^{\prime}$. 
Proof. Consider, first,

$$
\left|\left(S_{\tau}^{N} \psi-S_{\tau^{\prime}}^{N} \psi, \psi\right)\right| \leqq\left\|\left(S_{\tau}^{N}-S_{\tau^{\prime}}^{N}\right) \psi\right\|=Q_{N} .
$$

Then,

$$
Q_{N} \leqq\left\|\left(S_{\tau}-S_{\tau^{\prime}}\right) S_{\tau}^{N-1} \psi\right\|+\left\|S_{\tau}^{N-1} \psi-S_{\tau^{\prime}}^{N-1} \psi\right\|=\left\|\left(S_{\tau}-S_{\tau^{\prime}}\right) S_{\tau}^{N-1} \psi\right\|+Q_{N-1} .
$$

Now, let $\phi_{N}=S_{\tau}^{N-1} \psi$ :

$$
\begin{aligned}
\left\|\left(S_{\tau}-S_{\tau^{\prime}}\right) \phi_{N}\right\| & =\left\|\left(T_{\tau}-T_{\tau^{\prime}}\right) \phi_{N}\right\|=\left\|\sum_{n} c_{n}(N-1)\left(e^{-2 \pi i \tau n^{2}}-e^{-2 \pi i \tau^{\prime} n^{2}}\right) u_{n}(\theta)\right\| \\
& \leqq 2 \pi\left|\tau-\tau^{\prime}\right|\left(\sum_{n} n^{4}\left|c_{n}(N-1)\right|^{2}\right)^{1 / 2} .
\end{aligned}
$$

By Lemma 2, the term within brackets grows no faster than const $N^{4}$. Therefore, $Q_{N} \leqq \gamma\left|\tau-\tau^{\prime}\right| N^{2}+Q_{N-1}$ for a suitable $\gamma>0$. Since $Q_{0}=0$, we get $Q_{N} \leqq\left|\tau-\tau^{\prime}\right| \gamma N^{3}$. Now,

$$
\left|\mathscr{R}_{\tau}(N, \psi)-\mathscr{R}_{\tau^{\prime}}(N, \psi)\right| \leqq \frac{2}{N} \sum_{K=1}^{N} Q_{K} \leqq \frac{2}{N} \gamma\left|\tau-\tau^{\prime}\right| \sum_{K=1}^{N} K^{3} \leqq 2 \gamma\left|\tau-\tau^{\prime}\right| N^{3} .
$$

Theorem 2. For generic $V$ and for any given value of $E>0$ there is a nonempty set of irrationals $\tau \in(0,1)$ such that $\sigma_{E}$ is contained in the continuous subspace of $S_{\tau}$.

Proof. Let $V$ be generic in the sense of Theorem 1. For integer $\bar{q}$ and for a given $\varepsilon>0$, define $v_{E}(\bar{q}, \varepsilon)$ as the smallest integer such that

$$
\forall \psi \in \sigma_{E}, \forall \tau=p / q, q \leqq \bar{q}, p<q, \forall N \geqq v_{E}(\bar{q}, \varepsilon),\left|\mathscr{R}_{\tau}(N, \psi)\right|<\varepsilon .
$$

That such $v_{E}(\bar{q}, \varepsilon)$ exists is ensured by Lemma 1 . By its very definition, $v_{E}(\bar{q}, \varepsilon)$ is non-decreasing for $\bar{q} \rightarrow \infty$ and for $\varepsilon \rightarrow 0$. Then let $\sigma(x), \eta(x)$ be real valued functions of $x>0$, such that

$$
\sigma(x)>0, \eta(x)>0, \quad \text { and } \quad \sigma \rightarrow 0, \eta \rightarrow 0 \text { for } \quad x \rightarrow \infty .
$$

Define

$$
\phi_{E}(q)=\frac{\sigma(q)}{v_{E}^{3}(q, \eta(q))} .
$$

As $q \rightarrow \infty, \phi_{E}(q) \rightarrow 0$ by the definition of $\sigma, \eta, v$.

Now consider the nonempty set $L_{\phi_{E}} \subset[0,1]$ of irrationals $\bar{\tau}$ that can be obtained as the limit of a sequence $\tau_{n}=p_{n} / q_{n}$ of rationals in such a way that $\left|\bar{\tau}-\tau_{n}\right|<\phi_{E}\left(q_{n}\right)$. For any such $\bar{\tau}$, and for any entire $\psi \in \sigma_{E}$,

$$
\mathscr{R}_{\bar{\tau}}(N, \psi) \leqq\left|\mathscr{R}_{\bar{\tau}}(N, \psi)-\mathscr{R}_{\tau_{n}}(N, \psi)\right|+\mathscr{R}_{\tau_{n}}(N, \psi) \leqq \beta\left|\bar{\tau}-\tau_{n}\right| N^{3}+\mathscr{R}_{\tau_{n}}(N, \psi),
$$

thanks to Lemma 3. Now consider $N_{n}=v_{E}\left(q_{n}, \eta\left(q_{n}\right)\right)$. By the definition of $v_{E}$, $N_{n} \rightarrow \infty$ for $n \rightarrow \infty$. We have

$$
\mathscr{R}_{\bar{\tau}}\left(N_{n}, \psi\right) \leqq \beta\left|\bar{\tau}-\tau_{n}\right| v_{E}^{3}\left(q_{n}, \eta\left(q_{n}\right)\right)+\eta\left(q_{n}\right) \leqq \beta \sigma\left(q_{n}\right)+\eta\left(q_{n}\right) \rightarrow 0 \quad \text { for } \quad n \rightarrow \infty .
$$

On the other hand, we know that $\lim _{N \rightarrow \infty} \mathscr{R}_{\tau}(N, \psi)$ exists $\forall \tau, \forall \psi$, its value being $\int_{0}^{2 \pi} \mu(\{\theta\}) d \mu(\theta)$, where $d \mu$ is the spectral measure of $S_{\tau}$ relative to $\psi$. 
It follows that $\lim _{N \rightarrow \infty} \mathscr{R}_{\tilde{\tau}}(N, \psi)=0$; therefore, $\mu(\{\theta\})=0 \mu$-almost everywhere, i.e., $\mu$ is a purely continuous measure. Therefore, all entire $\psi \in \sigma_{E}$ belong to the continuous subspace of $S_{\bar{\tau}}$. By a density argument, the same holds for $\forall \psi \in \sigma_{E}$.

III.

The result of Theorem 2 above is certainly far from complete. At least two points need to be clarified:

1) How large is the set $L_{\phi_{E}}$ and how does its size depend on the energy $E$ and on the parameter $\mu$ ? If the quantum regime associated with values $\tau \in L_{\phi_{E}}$ is to correspond somehow to classical chaotic motion, then one expects the measure of $L_{\phi_{E}}$ to increase with $\mu$, tending eventually to 1 . To answer this question one needs more detailed information on the structure of the resonant spectra, in order to estimate $v_{E}(q, \varepsilon)$, and then $\phi_{E}$.

2) What is the nature of the continuous nonresonant spectrum? On account of numerical results obtained for the standard rotator $(V=\cos \theta)$ we expect the measure of the resonant spectrum to vanish in the limit $q \rightarrow \infty$, in that case, and in the generic case too. Then the nonresonant continuous spectrum would be singularcontinuous. We conjecture that quantum chaos in periodically perturbed quantum systems is connected with the occurrence of this particular type of quasi-energy spectra.

Both 1) and 2) are the object of current investigations. Nevertheless, an objection of a more general nature might be raised against our result.

In this, as well as in other cases in which "generic" results are established, one may be doubtful about the extent to which properties which are "generic" in a technical sense are actually to be observed in a majority of cases.

However necessary, this remark affects but marginally the relevance of our result. As a matter of fact, we now positively know that in some periodically perturbed quantum systems, there is room for a type of behaviour that is neither recurrent nor resonant, and strongly suspect that it is connected with the occurrence of a singular continuous quasi-energy spectrum. We think that this fact gives some firmer ground to investigations on the possibility of quantum chaotic behaviour. Indeed, it raises some interesting questions: how is this type of quantum motion related to classical chaos? Is it liable to experimental detection?

Certainly, we will not be hasty in dismissing it as a mathematical curiosity just on account of its unusual mathematical nature: after all, classical chaos itself may be elusive to an exceedingly square attitude.

\section{Appendix}

We prove here that the family of unitary matrices $S_{0}=S_{0}\left(V_{0}, V_{1}, \ldots, V_{q-1}\right)$ is generic in the sense that any degeneracy can be removed from any $S_{0}$ by an arbitrarily small variation of the $V_{i}$ 's. The characteristic polynomial $P\left(\lambda, V_{0}, \ldots, V_{q-1}\right)$ of $S_{0}$ has multiple roots, if and only if the resultant $G\left(V_{0}, \ldots, V_{q-1}\right)$ of $P$ and $P_{\lambda}^{\prime}$ vanishes ${ }^{[9]}$. $G$ is the determinant of a matrix whose elements are coefficients in $P$ or in $P_{\lambda}^{\prime}:$ hence, 
$G$ is an entire function of $V_{0}, \ldots, V_{q-1}$. There is at least one choice of $V_{0}, \ldots, V_{q-1}$ such that $S_{0}$ has no degenerate eigenvalues. This is $\bar{V}_{j}=\frac{2 \pi j}{\mu q},(j=0, \ldots, q-1)$. In fact, let $\alpha=\exp \left(\frac{2 \pi i}{q}\right)$. The circulant matrix $\Gamma$ has eigenvectors $\underline{u}_{j}(j=0, q-1)$ with components $\left(\underline{u}_{j}\right)_{n}=\alpha^{j n}$. Therefore, the matrix elements of $S_{0}\left(\bar{V}_{j}\right)$ over the base $\underline{u}_{j}$ are

$$
\left(S_{0}\right)_{j K}=v_{j} \delta_{j+1, K} \quad\left(S_{0}\right)_{q-1, K}=v_{q-1} \delta_{0, K},
$$

where $v_{j}$ are the eigenvalues of $\Gamma$. It is then easy to find that the eigenvalues of $S_{0}$ are the $q^{\text {th }}$ roots of $\prod_{j=0}^{q-1} v_{j}$; hence, they are distinct. Thus $G\left(\bar{V}_{0}, \ldots, \bar{V}_{q-1}\right) \neq 0$.

Now let $S_{0}$ have degenerate eigenvalues for $V_{j}=\overline{\bar{V}}_{j}$, so that $G\left(\overline{\bar{V}}_{0}, \ldots, \overline{\bar{V}}_{q-1}\right)$ $=0$. Find a $C^{q}$-valued analytic function $t \rightarrow V_{0}, \ldots, V_{q-1}$, such that $V_{j}(0)=\bar{V}_{j}$, $V_{j}(1)=\overline{\bar{V}}_{j} . G\left(V_{0}(t), \ldots, V_{q-1}(t)\right)$ will be an analytic function of $t$, not identically 0 : hence, $t=1$ must be an isolated zero, so that $G\left(V_{0}(t), \ldots, V_{q-1}(t)\right) \neq 0$ for $t$ arbitrarily near to 1 .

Acknowledgements. We thank B. Souillard and B.V. Chirikov for helpful discussions.

\section{References}

1. Casati, G., Chirikov, B.V., Izrailev, F.M., Ford, J.: In: Lecture Notes in Physics, Vol.93, Stochastic behaviour in classical and quantum Hamiltonian systems, Casati G., Ford J. Berlin, Heidelberg, New York: Springer 1979 (eds.).

2. Izrailev, F.M., Shepelyansky, D.L.: Quantum resonance for a rotator in a nonlinear periodic field. Theor. Mat. Fiz. 43, 417 (1980) [engl. transl. Theor. Math. Phys. 43, 553 (1980)]

3. Chirikov, B.V., Izrailev, F.M., Shepelyansky, D.L.: To be published

4. Shepelyansky, D.L.: Some statistical properties of simple classically stochastic quantum systems. Physica 8D, 208 (1983)

5. Hogg, T., Huberman, B.A.: Recurrence phenomena in quantum dynamics. Phys. Rev. Lett. 48, 711 (1982), and to be published

6. Grempel, D.R., Fishman, S., Prange, R.E.: Localization in an incommensurate potential: An exactly solvable model. Phys. Rev. Lett. 49, 833 (1982)

7. Dorizzi, B., Grammaticos, B., Pomeau, Y.: The periodically kicked rotator: recurrence and/or energy growth (preprint)

8. Donoghue, F.: Distributions and Fourier transforms. New York: Academic Press 1969

9. Van Waerden, L.: Algebra, p. 102. New York: Frederic-Hungar 1970

Communicated by Ya. G. Sinai

Received February 28, 1984; in revised form April 5, 1984 
